\title{
Nuclear Emulsion Readout Techniques Developed for the CHORUS Experiment
}

\author{
I. M. Papadopoulos \\ CERN, EP-CHE division, Geneva, Switzerland \\ CHORUS Collaboration
}

\begin{abstract}
The CHORUS [1] experiment is pursuing the study of the production and decay of short lived particles from neutrino interactions in a nuclear emulsion target. The extraction of the full information from the emulsion sheets has been possible only thanks to the development of fully automatic microscopes. The technique of automatic scanning, pioneered in Nagoya [2], involves precision mechanics, high quality optics and a readout scheme allowing for fast decisions. From the R\&D efforts within the various institutes of the CHORUS collaboration, the complementary approaches adopted by the Nagoya and CERN/NIKHEF groups are described here. Both are based on the principle that all information from the emulsion sheets should be extracted at the highest possible rate, limited only by the camera readout and the mechanical stability of the microscope stage.
\end{abstract}

\section{INTRODUCTION}

Nuclear emulsion is connected to many major discoveries in the early days of nuclear and particle physics. It still remains the detection technique with the best known three-dimensional spatial resolution, and zero intrinsic dead time. Its use has gradually decreased after the development of electronic detectors. Contrary to emulsion, electronic detectors offer immediate time correlated readout, and digitized output storable to computer accessible media. They serve therefore better the needs of most present experiments, which require large statistics of accumulated data for prompt and accurate physics results.

Recent developments in automatic emulsion scanning, have given a renaissance to emulsion as a particle detector. Automatic scanning allows for fast extraction of digital information from emulsion sheets, after they have been exposed to particle radiation. Not only does it make handling of large data sets possible, it also ensures that physics results can be produced shortly after the running of the experiment.

The CHORUS experiment is searching for $\nu_{\mu} \rightarrow \nu_{\tau}$ oscillations in the CERN-SPS wide band neutrino beam. The developments in the technique of automatic scanning allowed the experiment to extend its physics goals to the study of charm production by neutrinos.

Neutrino interactions take place in an $800 \mathrm{~kg}$ emulsion target, which is segmented in thin sheets oriented perpendicular to the incident neutrino beam. Each sheet has two layers of emulsion on a transparent plastic base. The thickness of the base and emulsion layers is $90 \mu \mathrm{m}$ and $350 \mu \mathrm{m}$ respectively. For the most downstream sheets, which serve as interface between the emulsion target and the electronic trackers, the thicknesses are $800 \mu \mathrm{m}$ and $100 \mu \mathrm{m}$ respectively.

The sensitivity of the emulsion is such that a minimum ionizing particle forms a latent image with 30 to 40 developed silver grains per $100 \mu \mathrm{m}$ along its trajectory. The intrinsic three-dimensional position resolution of the grains is about $0.2 \mu \mathrm{m}$. This allows the detection of $\nu_{\tau}$ charged current interactions, via the direct observation of the characteristic $\tau$ decay topology at a distance from the $\nu_{\tau}$ interaction vertex of order $1 \mathrm{~mm}$.

Electronic detectors located downstream the emulsion target are used to reconstruct the particle trajectories from neutrino interactions and to measure the relevant kinematical variables. They also assign a time stamp to the tracks of each event. The tracks have to be followed inside the emulsion target up to the primary interaction vertex.

The analysis of all CHORUS data has been possible only thanks to the construction of fully automatic microscopes, originally developed at the Nagoya University. In this paper the systems developed in Nagoya and at CERN are described. Emulsion scanning R\&D has also been performed in Naples, Münster and Salerno [3].

\section{The PRinciple of AUtomatic EMUlsion SCANNING}

Conceptually, a microscope for automatic emulsion scanning consists of a computer driven mechanical stage, the appropriate optical system, a photodetector -typically a CCD camera- and its associated readout. Digital images are analyzed for the recognition of the silver grains inside the developed emulsion gel.

A microscope view is a series of successive images taken along the beam direction (a microscope view). Grains belonging to different layers can be combined to form a straight line, whose parameters (slope and position) are compared to those of a track reconstructed by the electronic trackers.

In practice, the microscope scans some area around a. nominal position at the emulsion sheet, searching for straight tracks segments of a nominal slope. For the most downstream sheet these nominal values are given by the parameters of the tracks reconstructed in the electronic detectors. For all other sheets, the updated parameters from a segment found in the previously scanned sheet are used. The number of views required to complete the scanning of a prediction is determined not only by the prediction precision, which defines the area of emulsion surface to be covered, but also by the size of the 
camera sensitive area, and by the size of the field of view given by the optics.

Since the emulsion sheets are exposed perpendicular to the neutrino beam at the actual experiment, the tracks from neutrino interactions are oriented around the right angle to the sheet plane. All tracks within the angular acceptance of the CHORUS electronic detectors $(400 \mathrm{mrad})$ are therefore contained in a single microscope view.

Data taking of a view is performed with the stage driving the objective at a constant speed along the perpendicular to the emulsion sheet. At the same time the camera captures emulsion images at the objective's current focal position. The motor speed and the camera frame rate determine the spacing between successive planes. This spacing should be such that the information extracted from every plane is independent Thus it should not be smaller than the optical depth of focus.

Track reconstruction has to be performed while the stage moves to the central position of the next view, and completed before a new view can be acquired.

\section{ThE IMPLEMENTATION IN NAGOYA}

The microscopes developed in Nagoya [4] are based on a NIKON stage capable of handling emulsion sheets with dimension up to $36 \times 35 \mathrm{~cm}^{2}$. The three axes of the stage are driven by micro-stepper motors, and their position is read out by linear encoders. The position accuracy of the horizontal $\mathrm{X}, \mathrm{Y}$ axes and the vertical $\mathrm{Z}$ axis is $1 \mu \mathrm{m}$ and 0.25 $\mu \mathrm{m}$ respectively. The computer operating the microscope drives the stage through an ISA-based controller.

The NIKON optical system is fixed-focus, as the depth-focusing is done by moving the Z-stage, where the objective is mounted. The condenser height is tuned so that the light from the source is focused at a position in the middle of an emulsion sheet mounted on the stage. The optics houses a Tiyoda $50 \times$ oil immersion objective. It delivers a flat field with diameter of $200 \mu \mathrm{m}$, and depth of about $3 \mu \mathrm{m}$.

The light source is a 100 Watt Halogen lamp with an infrared blocking green filter. The light intensity is controlled by an analog signal fed to the lamp power supply, generated by an ISA-based DAC card. The purpose of the light control is to keep the same light conditions at both sides of an emulsion sheet, independent of the darkness of the emulsion.

The image collected by the objective is focused on a SONY CCD camera with 485 lines of 640 pixels each. It operates at $120 \mathrm{~Hz}$ and has an electronic shutter which leaves the active elements of the CCD under light exposure for $1 \mathrm{~ms}$. The readout is done via two separate ports, one for the even and one for the odd lines of the image. During the readout each port supplies $35 \mathrm{Mpixels} / \mathrm{s}$ as analog pulse-height information.

The emulsion readout is based on the Hardware Track Selector (TS) [5], a bus system equipped with a number of electronic modules responsible for both image analysis and track reconstruction. The TS is connected to the computer driving the microscope with its ISA bus.
A microscope view consists always of sixteen frames. The signal from each output port of the CCD camera is first amplified and then driven to an 8-bit FADC. In the digital image thus obtained, the grains in-focus are seen as small objects with high contrast to the background, typically occupying not more than three pixels along a line. To enhance the grains in the emulsion image, a high-pass digital FIR filter is applied along every line. After a threshold cut of the filter's 4-bit output, the pixels belonging to grains are identified and assigned a gray value of 1 , while the rest are reset to zero; hence a binarized image is formed. In the binarized image the grains are expanded by one or more pixels around their circumferences. This is done for the track finding to be less influenced by emulsion distortion and the physical grain dispersion along a particle trajectory. The binarized images are zero-suppressed and the positions (column, row) of the signal pixels are stored in FIFO buffers to be used in the next TS operation, the track finding. The treatment of a single emulsion image is completed within the $8 \mathrm{~ms}$ of the camera cycle.

The track finding procedure starts as soon as the output from the last image is available. If the binarized images are summed, after applying to each of them a shift proportional to the opposite of the nominal track angle, candidate tracks can be identified as pronounced clusters on the sum image. The summing is performed using processor elements based on FPGAs. The clustering on the sum image is done on a Pentium processor integrated in the same board as the rest of the processor elements.

For normal prediction scanning, covering $25 \mathrm{mrad}$ around the nominal slope, relatively few processor elements can try out all shifts in less than $300 \mathrm{~ms}$. If $40-50$ processor elements are used in parallel, up to $400 \mathrm{mrad}$ can be fully covered within the same time. This enables general scanning of all potentially interesting tracks within every microscope view, at a frequency limited only by the camera frame rate and the stage speed. Such a system is operational since the beginning of 1999 . It should be noted that the scanning speed of these system is almost 10 and 100 times larger than the ones available two and four years ago respectively. In the Nagoya emulsion laboratory all scanning stations are gradually upgraded to implement the latest version of the TS. These scanning stations are expected to offer the most significant contribution to the next phase of the CHORUS data analysis.

In summary, using $140 \times 160 \mu \mathrm{m}^{2}$ views, the TS technology and the light mechanics of the microscope stage and the optical system allow for real time general scanning of tracks with slopes up to $400 \mathrm{mrad}$ at a rate of $3 \mathrm{~Hz}$. Despite the hardware limit of the sixteen planes per view, the achievable measurement accuracy ensures high track finding and vertex reconstruction efficiencies.

\section{The Scanning Facility at CERN}

The first scanning facility at CERN [6] has been set up in mid 1996. The first microscope was very similar to the existing microscopes in Nagoya, which served as a training device to the scanning techniques. Since then the CERN and NIKHEF 
groups of CHORUS built another three scanning stations, made of completely new hardware elements. Moreover, new ideas in the scanning strategies have been applied.

The microscope stages were manufactured by MICOS AG [7]. They were designed to accommodate a full CHORUS sheet $\left(35 \times 70 \mathrm{~cm}^{2}\right)$ to reduce manipulation overhead. The three axes are driven by micro-stepper motors with a step size of $0.05 \mu \mathrm{m}$. Their position is continuously monitored by linear encoders which provide a resolution of $0.1 \mu \mathrm{m}$ and $0.25 \mu \mathrm{m}$ for the vertical and horizontal axes respectively. The controller of the stage communicates with the host computer through an RS232 interface.

In collaboration with Jenoptik AG, we designed an optical system to deliver a large field of view [8]. Using a $40 \times$ oil immersion objective the optics provide a flat view of 500 $\mu \mathrm{m}$ diameter with a focal depth below $1.5 \mu \mathrm{m}$. A mercury arc lamp and a package of spectral filters provide an almost monochromatic blue light source with a wavelength of 436 nm. Before the light reaches the condenser for the final focusing, it passes through a unit with two liquid crystal cells: a variable attenuator and a light shutter. The light attenuation is determined by an analog signal generated by a multiple channel DAC-ADC device [9], which in turn communicates with the host PC via RS232 signals. The shutter is synchronized with the camera, has a rise time of about $0.1 \mathrm{~ms}$, and attenuates the light by a factor of 10 when closed.

For the immersion oil, a containment system was developed at CERN to keep it always confined in a small region around the objective lens. The system is based on a ring embracing the objective with rubber edges, which ensure that the emulsion sheet below slides smoothly when the horizontal stage axes are moving. The oil circulates in a closed circuit including the ring itself, a pair of plastic tubes, an oil tank and a peristaltic pump [10].

The emulsion image is readout by a Thomson THX 788A camera [11]. It is built around a CCD chip with $1024 \times 1024$ useful square pixels with a size of $14 \times 14 \mu \mathrm{m}^{2}$ each. The camera can be read out at $30 \mathrm{~Hz}$ using two amplifiers which read half a line each. The camera kit includes a 12-bit ADC for each amplifier output, but it is the 8 least significant bits that are currently used to form the digital image. This choice was made to effectively compensate for the low quantum efficiency of the CCD at the blue light delivered by the optics. Apart from the $2 \times 12$ data signals, the camera also provides frame, line and pixel synchronization signals. A set of digital input channels can be used to set the camera's modes and to trigger the exposure. The exposure time itself is defined by the length of a digital pulse.

The flow of data and control signals is presented schematically in Figure 1. The data signals are transferred from an I/O driver card on the camera kit to an interface card close to the host PC, as LVDS. The control signals, whose integrity is less critical, are transferred with an RS422 signal. Among these digital control signals is the one which operates the shutter of the optical system.

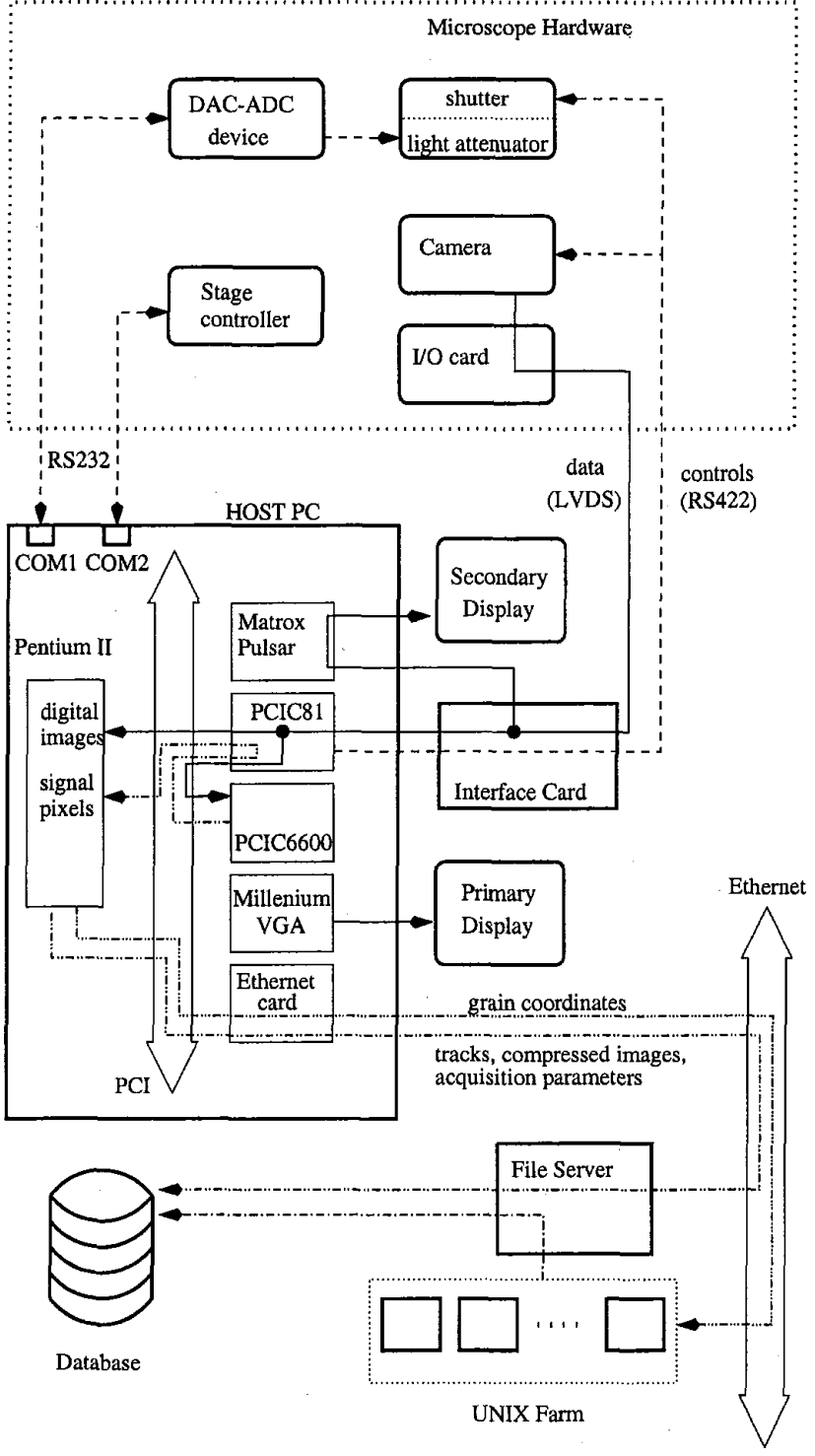

Figure 1: The flow of data, and control signals in the readout scheme of an automatic emulsion scanning microscope at CERN.

The interface card delivers the data to both the PCIC81 DSP card and the Matrox Pulsar frame grabber. The latter displays the raw emulsion image as video on a secondary monitor. It also provides graphical tools to monitor the scanning results.

The PCIC81 card manufactured by Blue Wave Systems [12] is the manager of the data acquisition cycle and the image processing. It is based on the Texas Instruments MVP TMS320C80 chip and includes a digital video interface. Its main function is to control the acquisition cycle by sending the appropriate signals to the camera and the shutter and by guiding the raw data from its input to the PCIC6600 card and the memory of the host PC. Prior to the introduction of the PCIC6600 cards in the systems, it was also used to analyze the images for grain identification, with the camera operating at lower frequency. 
The Blue Wave PCIC6600 board acts as a slave device of the TMS320C80 processor on the PCIC81 card. It has two TITMS320C620 DSPs, whose sole purpose is the application of a high-pass IIR filter on the digital image to identify the pixels belonging to grains. To make maximal use of the processor's capabilities, all time critical procedures itself have been coded in native assembly language. Once an image is analyzed the signal pixels are packed and sent back to the PCIC 81 card.

The acquisition cycle keeps running continuously on the DSP cards, independently of the host PC. For on-line track finding the Pentium processor in the $\mathrm{PC}$ requests from the PCIC81 the data of a number of successive images. This number may vary from 10 to 200 planes, depending on the type of scanning being performed. For normal prediction scanning a set of about 20 planes is used. The pixels are clustered into grains in the shadow of data taking, and while the stage moves to the next view an on-line tracking algorithm is performed to identify candidate tracks. Those candidates are stored in an Objectivity database together with all data taking parameters.

The flexibility offered by the use of DSP boards allows for data taking along the whole depth of the emulsion sheet, using the maximum number of planes. This is the case for example when there is a strong signal for the existence of an interaction vertex, or of a decay topology. Instead of performing on-line tracking on the data acquisition $\mathrm{PC}$, the positions of the identified grains are sent via the network to a UNIX farm, performing track and vertex finding on the events they receive from the microscopes. The output of this general tracking procedure is stored in the same database as the output from the on-line tracking in prediction scanning. In special cases the raw data is stored together with the filtered output. The digital images are JPEG compressed and written to the database, to be used later in interactive checks off-line.

Thanks to the optics performance and camera size, the drawbacks of the low camera frequency and heavy mechanics are compensated by the large volume of emulsion in a single view. Using parallel processing in the general tracking, performances similar to the Nagoya TS are reached in an entirely different way.

\section{CONCLUSIONS}

The two different approaches adopted by Nagoya and CERN in automatic scanning lead to complementary advantages and disadvantages in the various steps of the scanning procedure.

The implementation in Nagoya is based on the consideration that an emulsion sheet is a storage medium itself. The microscopes act as data processors whose output is directly a set of reconstructed track segments. To achieve rapid scanning they feature light mechanics and hardwired image processing logic in embedded electronics modules.

At CERN, the information which is extracted from the emulsion sheets, is the position of the developed silver grains. The reconstruction of track segments is performed in software, either on-line during the scanning, or off-line once the grain positions have been stored in the database. The second method allows the repeating of the track finding procedure as soon as new pattern recognition and reconstruction algorithms have been developed, without the need of rescanning the emulsion sheets.

In conclusion, the Nagoya implementation is aiming at high scanning speed, while the CERN one at high flexibility. Their overall performance is similar and the combination of these techniques and the ones developed in the other scanning laboratories of CHORUS, ensure that the full information from the CHORUS emulsion target will be available for physics analysis.

\section{ACKNOWLEDGMENTS}

I would like to thank the colleagues from the Nagoya FKEN laboratory, especially K. Niwa, T. Nakano, and K. Kodama for the many fruitful discussions on the Track Selector operation.

The setup of the CERN-NIKHEF scanning facility was realized only thanks to the close collaboration of physicists and engineers from the CERN, NIKHEF and Brussels groups of CHORUS: J. Panman, B. Van de Vyver, J. Uiterwijk, E. Pesen, O. Melzer, M. Chizchov, M. Doucet, B. Friend, H. Meinhard, P. Zucchelli, G. Grégoire, W. Flegel, J-P. Dupraz, J-P. Fabre, G. Van Beek, and D. Bourillot.

Finally, I would like to thank P. Strolin and R. van Dantzig for their comments on the manuscript.

\section{REFERENCES}

[1] E. Eskut et al. "The CHORUS experiment to search for $\nu_{\mu} \rightarrow \nu_{\tau}$ oscillation" Nucl. Instr. Meth., vol. A40I, 1997 pp. $7-44$

[2] S. Aoki et al. "Fully Automated Emulsion Analysis System", Nucl. Instr. Meth., vol. B51, 1990 pp. 446-472

[3] S. Amendola et al. "SySal: System of Salerno" hepex/9901031, 1999 1st International Workshop on Nuclear Emulsion Techniques, Nagoya, Japan, 12-14 June 1998.

[4] http:://flab.phys.nagoya-u.ac.jp/flab.html

[5] T. Nakano Ph.D. thesis, Nagoya Univ., Japan, 1997

[6] http:://choruswww.cern.ch/CERN-Microscope

[7] Micos GmbH D-79224 Umkirch, Germany.

[8] K. Winkler et al. "Optical microscope for nuclear emulsion readout- system design and results in application" Proc. SPIE 44th annual meeting, paper 3779-43, 1999.

[9] CerealBox LV824-H, manufactured by BG SYSTEMS INC., Palo Alto, CA 94306, USA.

[10] MasterFlex $\mathrm{C} / \mathrm{L}$ model 77120-62, manufactured by ColeParmer Instrument Company, Illinois 60061, USA.

[11] Thomson THX 788A CCD, manufactured by THOMSON-CSF Semiconucteurs Specifiques, F-38521, Saint Egreve, France.

[12] BLUE WAVE SYSTEMS INC., 2410 Luna Road, Carrollton, TX 75006, UK. 